\title{
Liquid-like Layers on Ice in the Environment: Bridging the Quasi-liquid and Brine Layer Paradigms SUPPLEMENTARY MATERIAL
}

\author{
Min H. Kuo, Samar G. Moussa, V. Faye McNeill
}

Department of Chemical Engineering, Columbia University, New York, New York 10027

*Author to whom correspondence should be addressed: vfm2103@columbia.edu; phone (212) 854-2869; FAX (212) 854-3054

\section{A. Derivation of BL Model (eqs. 1 and 2)}

\section{List of symbols}

$\begin{array}{ll}\text { Symbol } & \text { Quantity } \\ T & \text { System temperature } \\ T_{m} & \text { Bulk melting temperature of ice } \\ P & \text { System pressure } \\ f_{w}^{i c e} & \text { Fugacity of pure ice } \\ \hat{f}_{w}^{b r i n e} & \text { Fugacity of water in brine layer } \\ X_{w} & \text { Mole fraction of water in brine layer } \\ X_{w, 0} & \text { Mole fraction of water in unfrozen solution } \\ H_{w}^{\text {ice }} & \text { Enthalpy of ice at T, P } \\ H_{w}^{i c e, 0} & \text { Enthalpy of ice at reference state } \\ \bar{H}_{w}^{\text {brine }} & \text { Partial molar enthalpy of water in brine layer } \\ H_{w}^{\text {liq,0 }} & \text { Enthalpy of water in unfrozen liquid solution } \\ \bar{V}_{w}^{b r i n e} & \text { Partial molar volume of water in brine layer } \\ \Delta H_{w}^{\text {fus }} & \text { Enthalpy change of fusion } \\ \Delta \bar{H}_{w}^{\text {brine }} & \text { Enthalpy change upon formation of brine layer } \\ \Delta V_{w}^{\text {fus }} & \text { Volume change of fusion } \\ \Delta \bar{V}_{w}^{\text {brine }} & \text { Volume change upon formation of brine layer } \\ \gamma_{w} & \text { Activity coefficient of water in brine }\end{array}$


$f_{w}^{v a p} \quad$ Fugacity of water in vapor space above ice

$\Delta H_{w}^{v a p} \quad$ Enthalpy change upon vaporization

$\Delta V_{w}^{v a p} \quad$ Volume change upon vaporization

$\varphi \quad$ Liquid water fraction

$n_{w}^{\text {brine }} \quad$ Moles of water in brine layer

$n_{w} \quad$ Total number of moles of water

$n_{s} \quad$ Number of moles of solute

$d \quad$ Thickness of liquid layer

$V \quad$ Volume of ice sample

A Surface area of ice sample

$\rho_{w} \quad$ Density of water

$\rho_{\text {ice }} \quad$ Density of ice

At equilibrium (Tester and Modell, 1996),

$$
d \ln f_{w}^{i c e}=d \ln \hat{f}_{w}^{b r i n e}
$$

Expanding,

$\left(\frac{\partial \ln f_{w}^{i c e}}{\partial T}\right)_{P} d T+\left(\frac{\partial \ln f_{w}^{i c e}}{\partial P}\right)_{T} d P=\left(\frac{\partial \ln \hat{f}_{w}^{\text {brine }}}{\partial T}\right)_{P, x_{w}} d T+\left(\frac{\partial \ln \hat{f}_{w}^{\text {brine }}}{\partial P}\right)_{T, x_{w}} d P+\left(\frac{\partial \ln \hat{f}_{w}^{\text {brine }}}{\partial x_{w}}\right)_{T, P} d x_{w}(\mathrm{~S} 2)$

Substituting for the partial derivatives of fugacity,

$$
-\left(\frac{H_{W}^{i c e}-H_{w}^{i c e, 0}}{R T^{2}}\right) d T+\frac{V_{W}^{i c e}}{R T} d P=-\left(\frac{\bar{H}_{W}^{\text {brine }}-H_{w}^{l i q, 0}}{R T^{2}}\right) d T+\frac{\bar{V}_{W}^{\text {brine }}}{R T} d P+\left(\frac{\partial \ln \hat{f}_{W}^{\text {brine }}}{\partial x}\right)_{T, P} d x_{W}
$$

Collecting terms,

$$
\begin{gathered}
\left(\frac{\partial \ln \hat{f}_{w}^{b r i n e}}{\partial x_{w}}\right)_{T, P} \frac{d x_{w}}{d T}=-\left(\frac{H_{w}^{i c e}-\bar{H}_{w}^{b r i n e}}{R T^{2}}\right)+\frac{V_{w}^{i c e}-\bar{V}_{w}^{\text {brine }}}{R T} \frac{d P}{d T} \\
\left(\frac{\partial \ln \hat{f}_{w}^{\text {brine }}}{\partial x_{w}}\right)_{T, P} \frac{d x_{w}}{d T}=-\left(\frac{\Delta H_{w}^{f u s}-\Delta \bar{H}_{w}^{b r i n e}}{R T^{2}}\right)+\left(\frac{\Delta V_{w}^{f u s}-\Delta \bar{V}_{w}^{b r i n e}}{R T}\right) \frac{d P}{d T}
\end{gathered}
$$

we know

$$
\hat{f}_{w}^{b r i n e}=\gamma_{w} f_{w} x_{w}
$$

therefore, 


$$
\left(\frac{\partial \ln \hat{f}_{w}^{b r i n e}}{\partial x_{w}}\right)_{T, P}=\frac{1}{\gamma_{w} f_{w} x_{w}} \gamma_{w} f_{w}=\frac{1}{x_{w}}
$$

and

$$
\frac{1}{x_{w}} \frac{d x_{w}}{d T}=\frac{d \ln x_{w}}{d T}=-\left(\frac{\Delta H_{w}^{f u s}-\Delta \bar{H}_{w}^{\text {brine }}}{R T^{2}}\right)+\left(\frac{\Delta V_{w}^{\text {fus }}-\Delta \bar{V}_{w}^{\text {brine }}}{R T}\right) \frac{d P}{d T}
$$

Also,

$$
\begin{gathered}
d \ln f_{w}^{v a p}=d \ln \hat{f}_{w}^{b r i n e} \\
\left(\frac{\partial \ln \hat{f}_{w}^{b r i n e}}{\partial x_{w}}\right)_{T, P} \frac{d x_{w}}{d T}=-\left(\frac{\Delta H_{w}^{v a p}-\Delta \bar{H}_{w}^{\text {brine }}}{R T^{2}}\right)+\left(\frac{\Delta V_{w}^{v a p}-\Delta \bar{V}_{w}^{b r i n e}}{R T}\right) \frac{d P}{d T}
\end{gathered}
$$

Equating eqs (S5) and (S10),

$$
\frac{d P}{d T}=\left(\frac{\Delta H_{w}^{v a p}-\Delta H_{w}^{f u s}}{T\left(\Delta V_{w}^{v a p}-\Delta V_{w}^{f u s}\right)}\right)
$$

Substituting eq (S11) into eq (S5),

$$
\frac{d \ln x_{w}}{d T}=-\left(\frac{\Delta H_{w}^{f u s}-\Delta \bar{H}_{w}^{\text {brine }}}{R T^{2}}\right)+\left(\frac{\Delta V_{w}^{f u s}-\Delta \bar{V}_{w}^{b r i n e}}{R T}\right)\left(\frac{\Delta H_{w}^{v a p}-\Delta H_{w}^{f u s}}{T\left(\Delta V_{w}^{v a p}-\Delta V_{w}^{f u s}\right)}\right)
$$

Neglect the partial molar enthalpy and volume of mixing, and simplify.

$$
\frac{d \ln x_{w}}{d T}=\frac{\Delta V_{w}^{f u s} \Delta H_{w}^{v a p}-\Delta H_{w}^{f u s} \Delta V_{w}^{v a p}}{R T^{2}\left(\Delta V_{w}^{v a p}-\Delta V_{w}^{f u s}\right)}
$$

Dividing through on top \& bottom by $\Delta \mathrm{V}_{\mathrm{w}}{ }^{\text {vap }}$ and using the fact that $\Delta \mathrm{V}_{\mathrm{w}}{ }^{\text {fus }}<<\Delta \mathrm{V}_{\mathrm{w}}$ vap, eq (S13) simplifies to:

$$
\frac{d \ln x_{w}}{d T}=\frac{-\Delta H_{w}^{f u s}}{R T^{2}}
$$

Integrating,

$$
x_{w}=x_{w, 0} \exp \left[-\frac{\Delta H_{w}^{f u s}}{R}\left(\frac{1}{T}-\frac{1}{T_{m}}\right)\right]
$$

In order to calculate layer thickness we first relate $\mathrm{x}_{\mathrm{w}}$ to the liquid water fraction, $\varphi$

$$
\varphi=\frac{n_{w}^{\text {brine }}}{n_{w}}=\frac{d A}{V} \frac{\rho_{\text {ice }}}{\rho_{w}}
$$

Where $d$ is BL thickness, $A$ and $V$ are dimensions of the sample, and $\rho$ is density. 
From the definition of $x_{w}$, we get

$$
x_{w, 0}=\frac{n_{w}}{n_{w}+n_{s}} \quad x_{w}=\frac{n_{w}^{\text {brine }}}{n_{w}^{\text {brine }}+n_{s}}
$$

Rearranging and substituting, we get

$$
d=\frac{V}{A} \frac{\rho_{w}}{\rho_{\text {ice }}}\left(\frac{x_{w}}{x_{w, 0}} \frac{\left(1-x_{w, 0}\right)}{\left(1-x_{w}\right)}\right)
$$

\section{B. Derivation of Semi-Empirical Models for the QLL}

Table 1: The semi-empirical models $\left(d_{Q L L, I n}, d_{Q L L,-1 / 2}, d_{Q L L,-1 / 3}\right)$ presented in section 3 of the

\begin{tabular}{|c|c|c|c|c|c|c|c|c|c|}
\hline & \multicolumn{3}{|c|}{$\left.\mid \ln \left(\left(T_{m}-T\right) / T_{m}\right)\right) \mid$} & \multicolumn{3}{|c|}{$\left(T_{m}-T\right)^{-1 / 2}$} & \multicolumn{3}{|c|}{$\left(T_{m}-T\right)^{-1 / 3}$} \\
\hline & Slope & Intercept & $\mathrm{R}^{2}$ & Slope & Intercept & $\overline{\mathrm{R}^{2}}$ & Slope & Intercept & $\mathrm{R}^{2}$ \\
\hline Dosch & 16.371 & -51.919 & 0.8218 & 66.855 & -19.83 & 0.9341 & 80.95 & -36.155 & 0.9037 \\
\hline Doppenschmidt & 10.262 & -17.72 & 0.8208 & 45.757 & 0.0502 & 0.8572 & 53.9 & 10.051 & 0.8521 \\
\hline Mazzega & 1.1 & -2.4711 & 1 & 0.8906 & 0.9297 & 0.6744 & 2.2058 & 0.1019 & 0.8231 \\
\hline Bluhm & 0.8423 & -2.1323 & 0.9165 & 3.7415 & -0.6945 & 0.9547 & 4.4728 & -1.5471 & 0.9596 \\
\hline Pittenger & 0.3151 & -0.8009 & 0.8919 & 1.2272 & -0.1601 & 0.9819 & 1.512 & -0.4723 & 0.9613 \\
\hline Sadtchenko & 2.4478 & -8.231 & 0.9786 & 2.7037 & 0.8935 & 0.974 & 5.3146 & -1.3638 & 0.9931 \\
\hline$?^{2}$-weighted avg & 4.866871 & -12.9671 & & 20.40124 & -3.31141 & & 24.01679 & -4.97332 & \\
\hline
\end{tabular}
manuscript obtained based on the fit parameter data.

\section{REFERENCES}

Bluhm, H., Ogletree, D. F., Fadley, C. S., Hussain, Z., and Salmeron, M.: The Premelting of Ice Studied With Photoelectron Spectroscopy, J. Phys.: Cond. Matter, 14 (8), L227-L233, 2002.

Doppenschmidt, A. and Butt, H.-J.: Measuring the Thickness of the Liquid-like Layer on Ice Surfaces with Atomic Force Microscopy, Langmuir, 16 (16), 6709-6714, 2000.

Dosch, H., Lied, A., and Bilgram, J. H.: Disruption of the Hydrogen-bonding Network at the Surface of Ih Ice Near Surface Premelting, Surf. Sci., 366 (1), 43-50, 1996.

Mazzega, E., Pennino, U. D., Loria, A., and Mantovani, S.: Volta Effect and Liquid-Like Layer at Ice Surface, J. Chem. Phys., 64 (3), 1028-1031, 1976. 
Pittenger, B., Fain, S. C., Cochran, M. J., Donev, J. M. K., Robertson, B. E., Szuchmacher, A., and Overney, R. M.: Premelting at Ice-Solid Interfaces Studied via Velocity-Dependent Indentation with Force Microscope Tips, Phys. Rev. B, 6313 (13), 134102, 2001.

Sadtchenko, V. and Ewing, G. E.: Interfacial Melting of Thin Ice Films: An Infrared Study, J. Chem. Phys., 116 (11), 4686-4697, 2002.

Tester, J.W. and Modell, M. Thermodynamics and its Applications, $3^{\text {rd }}$ Ed. Prentice Hall, Upper Saddle River, N.J. (1996) 